\title{
Geometric Combinatorics
}

\author{
$\operatorname{AUTHOR}(\mathrm{S})$ :
}

ASCHBACHER, MICHAEL

\section{CITATION:}

ASCHBACHER, MICHAEL. Geometric Combinatorics. 数理解析研究所講 究録 1995, 896: 32-37

\section{ISSUE DATE:}

1995-01

URL:

http://hdl.handle.net/2433/84459

RIGHT: 


\section{Geometric Combinatorics \\ Michael Aschbacher \\ California Institute of Technology}

I like to use the term geometric combinatorics to describe those areas of mathematics which study combinatorial objects possessing some geometric flavor. Examples of such objects include

Projective Planes.

Buildings.

Geometries in the sense of Tits.

Certain graphs.

Abstract simplicial complexes.

All these examples can be subsumed within the last two examples, which are closely related. Thus I will concentrate on graphs and simplicial complexes.

To provide some focus, I will talk about one particular application of geometric combinatorics in finite group theory. But I hope this discussion will illuminate some fairly general concepts.

In the application I will discuss, one seeks to prove the uniqueness of the sporadic groups subject to the hypothesis that the group possesses certain subgroups. The technique can also be used to establish presentations for the group. The approach involves associating to the group a graph or simplicial complex. The key step is to show the graph or complex is simply connected.

Now some details. We begin with some examples.

Examples (1) Let $\Gamma$ be a (undirected) graph. The clique complex of $\Gamma$ is the simplicial complex $K(\Gamma)$ with vertex set $\Gamma$ and simplices the finite cliques of $\Gamma$. Coversely if $L$ is a simplicial complex the graph $\Delta(L)$ of $L$ is the graph whose vertices are the vertices of $L$ and with $x$ adjacent to $y$ if $\{x, y\}$ is a simplex of $L$. Notice $L$ is a subcomplex of $K(\Delta(L))$ and $\Gamma=\Delta(K(\Gamma))$.

(2) Let $G$ be a group, $\mathcal{F}=\left(G_{i}: i \in I\right)$ a finite family of subgroups of $G$, and $\mathcal{C}(G, \mathcal{F})$ the simplicial complex with vertex set $\coprod_{i} G / G_{i}$ and with simplices the sets $s$ of vertices such that $\bigcap_{C \in s} C \neq \varnothing$. The complex $\mathcal{C}(G, \mathcal{F})$ is the coset complex of $G$ and $\mathcal{F}$. Notice $G$ is represented as a group of automorphisms on $\mathcal{C}(G, \mathcal{F})$ by right multiplication.

(3) Let $H$ be a subgroup of $G$ and $\theta$ a selfpaired orbital of $G$ on $G / H$. Then the graph of $H, \theta$ is the graph with vertex set $G / H$ and edge set $\theta$. Again $G$ is represented as a group of automorphisms on this graph by right multiplication.

This work was partially supported by NSF DMS-9101237 
Simple Connectivity. Let $C$ be a category and $X$ an object in $C$. A covering of $X$ in the category $C$ is a surjective local isomorphism $\alpha: \tilde{X} \rightarrow X$. Further a connected object $X$ is simply connected if it possesses no proper connected coverings. Of course one must decide what is meant by the terms "surjection", "local isomorphism", and "connected".

Example (4) If $\Gamma$ is a graph then a morphism $\alpha: \tilde{\Gamma} \rightarrow \Gamma$ is surjective if it is surjective on vertices and a local isomorphism if for each vertex $x$ of $\tilde{\Gamma}$, the restriction

$$
\alpha_{x}: x^{\perp} \rightarrow x \alpha^{\perp}
$$

is an isomorphism of graphs, where $x^{\perp}$ consists of $x$ together with all vertices adjacent to $x$.

(5) If $K$ is a simplicial complex then a simplicial map $\alpha: \tilde{K} \rightarrow K$ is surjective if it is surjective on vertices and a local isomorphism if for each vertex $x$ of $\tilde{K}$,

$$
\alpha_{x}: s t_{\tilde{K}}(x) \rightarrow s t_{K}(x \alpha)
$$

is an isommorphism of simplicial complexes, where $s t_{\tilde{K}}(x)$ consists of all simplices $s$ such that $s \cup\{x\}$ is a simplex.

Remarks (1) A morphism $\alpha: \tilde{\Gamma} \rightarrow \Gamma$ of graphs is a covering if and only if $\alpha$ : $K(\tilde{\Gamma}) \rightarrow K(\Gamma)$ is a covering of simplicial complexes.

(2) A simplicial map $\alpha: \tilde{L} \rightarrow L$ is a covering if and only if $\alpha: \Delta(\tilde{L}) \rightarrow \Delta(L)$ is a local bijection and $\Sigma(x) \alpha=\Sigma(x \alpha)$ for each vertex $x$ of $\tilde{L}$, where $\Sigma(x)$ is the set of simplices in $s t_{\tilde{L}}(x)$.

In particular both types of coverings can be studied using the following construction which is discussed in [AS] and [A2]. Let $\Delta$ be a graph and $P$ the set of paths $p=x_{0} \cdots x_{n}$ in $\Delta$. For $q=y_{0} \cdots y_{m} \in P$ with $y_{0}=x_{n}$ define

$$
\begin{aligned}
p q & =x_{0} \cdots x_{n} y_{1} \cdots y_{m} \\
p^{-1} & =x_{n} \cdots x_{0}
\end{aligned}
$$

Write $\operatorname{org}(p), \operatorname{end}(p)$ for the origin $x_{0}$ and end $x_{n}$ of $p$, respectively.

Define a set $C$ of cycles of $\Delta$ to be closed if it satisfies the following six properties:

(C1) $r r^{-1} \in C$ for all $r \in P$.

(C2) If $p \in C$ then $p^{-1} \in C$.

(C3) If $p, q \in C$ and $\operatorname{org}(p)=\operatorname{org}(q)$, then $p q \in C$.

(C4) If $p \in C$ then $r^{-1} p r \in C$ for each $r \in P$ with $\operatorname{org}(r)=e n d(p)$.

(C5) If $p$ is a cycle and $r \in P$ with $\operatorname{or} g(p)=e n d(r)$ and $r^{-1} r p \in C$, then $p \in C$.

(C6) $x x \in C$ for all $x \in \Delta$. 
Now $C$ defines an equivalence relation $\sim$ on $P$ by $p \sim q$ iff $\operatorname{org}(p)=\operatorname{org}(q)$, $\operatorname{end}(p)=\operatorname{end}(q)$, and $p q^{-1} \in C$. Such equivalence relations have nice properties. In particular if $\pi\left(\Delta, x_{0}\right)$ is the set of all cycles with origin $x_{0}$ then $\pi\left(\Delta, x_{0}\right) / \sim=$ $\tilde{\pi}\left(\Delta, x_{0}\right)$ is a group. Further if $\Phi\left(\Delta, x_{0}\right)$ denotes the set of paths with origin $x_{0}$ and $\tilde{\Phi}\left(\Delta, x_{0}\right)=\Phi\left(\Delta, x_{0}\right) / \sim$ then $\tilde{\Phi}\left(\Delta, x_{0}\right)$ is a graph with $\tilde{p}$ adjacent to $\widetilde{p x}$ for $x \in x_{n}^{\perp}$,

$$
\begin{aligned}
\operatorname{end}_{C}: \tilde{\Phi}\left(\Delta, x_{0}\right) & \rightarrow \Delta \\
\tilde{p} & \mapsto \operatorname{end}(p)
\end{aligned}
$$

is a local bijection of $\Delta$, and $\tilde{\pi}\left(\Gamma, x_{0}\right)$ acts regularly on the fibers of end via left multiplication. Further end $C$ is a covering if and only if $C$ contains all triangles of $\Delta$, and if $\mathcal{C}_{3}(\Delta)$ is the closed set generated by all triangles of $\Delta$ then $\operatorname{end}_{\mathcal{C}_{3}(\Delta)}$ is the universal covering of $\Delta$. Therefore:

Lemma. A connected graph $\Delta$ is simply connected if and only if $\mathcal{C}_{3}(\Delta)$ is the set of all cycles of $\Delta$. ( $\Delta$ is triangulable.) Further the fundamental group of $\Delta$ is $\pi_{1}(\Delta)=\tilde{\pi}\left(\Delta, x_{0}\right)$ and is regular on the fibers of the universal covering of $\Delta$.

Remarks (3) This is essentially the standard construction of the fundamental groupoid and the edge path group in combinatorial topology.

(4) The same formalism can be applied to a simplicial complex $K$ by taking $\Delta$ to be the graph $\Delta(K)$ of $K$. In this case the closed set $C$ determining the universal covering of $K$ is the one generated by all triangles of $\Delta$ which are simplices of $K$.

(5) The formalism together with some simple minded observations supplies a method for calculating when $\Delta$ is simply connected. Namely determine if $\Delta$ is triangulable. With enough information about $\Delta$ this method is effective.

(6) If $\Delta$ is not nice, stronger techniques are needed to prove $\Delta$ is simply connected. Algebraic topology supplies some techniques, but these too are of restricted utility in many interesting combinatorial situations.

Problem 1. Generate general techniques for proving graphs and simplicial complexes are simply connected.

Group Amalgams. Let $I=\{1, \ldots, n\}$ be a set of finite order $n$. An amalgam of rank $n$ is a family

$$
A=\left(\alpha_{J, K}: P_{J} \rightarrow P_{K}: J \subset K \subset I\right)
$$

of group homomorphisms such that for all $J \subset K \subset L, \alpha_{J, K} \alpha_{K, L}=\alpha_{J, L}$. We will only consider amalgams where the maps $\alpha_{J, K}$ are injections. 
Example (6) Let $\mathcal{F}=\left(G_{i}: i \in I\right)$ be a family of subgroups of a group $G$. For $J \subseteq I$ let $J^{\prime}=I-J$ be the complement to $J$ in $I$ and define $G_{J}=\bigcap_{j \in J} G_{j}$. Define $P_{J}=G_{J^{\prime}}$. Thus $P_{J} \cap P_{K}=G_{J^{\prime}} \cap G_{K^{\prime}}=G_{J^{\prime} \cup K^{\prime}}=G_{(J \cap K)^{\prime}}=P_{J \cap K}$. Also for $J \subset K \subset I$, define $\alpha_{J K}: P_{J} \rightarrow P_{K}$ to be inclusion. Then

$$
\mathcal{A}(\mathcal{F})=\left(\alpha_{J K}: P_{J} \rightarrow P_{K}: J \subset K \subset I\right)
$$

is an amalgam.

A morphism $\phi: A \rightarrow \bar{A}$ of rank $n$ amalgams is a family

$$
\phi=\left(\phi_{J}: P_{J} \rightarrow \bar{P}_{J}: J \subset I\right)
$$

of group homomorphisms such that for all $J \subset K \subset I$ the obvious diagram commutes:

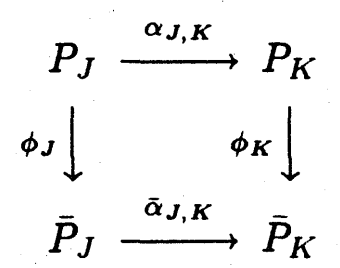

A completion $\beta: A \rightarrow G$ for $A$ is a family $\beta=\left(\beta_{J}: P_{J} \rightarrow G\right)$ of group homomorphisms such that $G=\left\langle P_{J} \beta_{J}: J \subset I\right\rangle$ and for all $J \subset K \subset I$ the obvious diagram commutes:

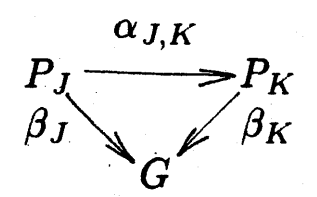

The completion $\beta: A \rightarrow G$ is said to be faithful if each $\beta_{J}$ is an injection.

There exists a universal completion $\iota: \mathcal{A} \rightarrow G(\mathcal{A})$ of an amalgam $\mathcal{A}$. Namely $G(A)$ is the free product of the groups $P_{J}, J \subseteq I$, modulo the relations induced by the maps $\alpha_{J, K}$ with $\iota$ the induced map.

Assume $\beta: \mathcal{A} \rightarrow G$ is a faithful completion. Identifying $P_{J}$ with its image $G_{J^{\prime}}=P_{J} \beta$ in $G$ we can think of $\beta$ as inclusion and $G$ as arising in Example 6. We can then associate the simplicial complex $\mathcal{C}(G, \mathcal{F})$ to $\mathcal{A}$. This complex is connected as $G=\langle\mathcal{F}\rangle$. We also have our universal completion $\tilde{G}=G(\mathcal{A})$ and its complex $\mathcal{C}(\tilde{G}, \tilde{\mathcal{F}})$. By the universal property of $\tilde{G}$ there is a surjective group homomorphism $\alpha: \tilde{G} \rightarrow G$ which is a local isomorphism in the sense that the restriction $\alpha_{J}: \tilde{G}_{J} \rightarrow G_{J}$ of $\alpha$ to $\tilde{G}_{J}$ is an isomorphism for each $J \subseteq I$, and this implies that the induced map $\alpha: \mathcal{C}(\tilde{G}, \tilde{\mathcal{F}}) \rightarrow \mathcal{C}(G, \mathcal{F})$ is a covering of simplicial complexes. In particular if $\mathcal{C}(G, \mathcal{F})$ is simply connected then this covering is an isomorphism, which in turn shows $\alpha: \tilde{G} \rightarrow G$ is an isomorphism. This gives us a 
presentation for $G$ as the free product of groups $G_{J}$ modulo the relations supplied by the amalgam. Further if $G$ is simple, it gives us a characterization of $G$ as the unique nontrivial connected completion of $\mathcal{A}$. The critical missing piece of information here is of course the proof that $\mathcal{C}(G, \mathcal{F})$ is simply connected.

We now use this point of view to study the sporadic simple groups.

Uniqueness of the Sporadic Groups. One important step in the Classification of the finite simple groups is to prove each sporadic group is unique subject to some suitable hypothesis. The appropriate hypotheses are restrictions on the centralizer of some involution. For example for half of the sporadics, the following hypothesis seems to provide the best characterization. Let $L$ be a group and $n$ a positive integer.

Hypothesis $\mathcal{H}(w, L) . G$ is a finite group, $z$ is an involution in $G, H=C_{G}(z)$, and $Q=F^{*}\left(C_{G}(z)\right)$. Assume $Q$ is an extraspecial 2-group of order $2^{2 w+1}, H / Q \cong L$, and $z$ is not weakly closed in $Q$ with respect to $G$.

For example the Monster is the unique group $G$ satisfying Hypothesis $\mathcal{H}\left(12, C o_{1}\right)$. The existing treatment of the existence, uniqueness, and basic structure of the sporadics in the literature is inadequate for a number of reasons. Thus for a several years I have been engaged in a program to produce a uniform, self-contained, and fairly simple and elegant second generation treatment of these foundational properties of the sporadics. My approach to uniqueness uses the idea outlined above; it was developed in collaboration with Yoav Segev.

Assume we wish to prove that a sporadic is characterized by some hypthesis $\mathcal{H}$ like the one described above; that is we wish to show that up to isomorphism there is at most one group satisfying $\mathcal{H}$. One must:

(i) Prove that if $G$ is a group satisfying hypothesis $\mathcal{H}$ then $G$ is simple and there exists a suitable family $\mathcal{F}$ of subgroups such that

(ii) the amalgam $\mathcal{A}(\mathcal{F})$ is determined up to isomorphism by $\mathcal{H}$ independent of $G$, and

(iii) $\mathcal{C}(G, \mathcal{F})$ is simply connected.

There are often technical difficulties involved with step (i), but they have been well understood for some time. In [AS], Segev and I prove some theorems which can be used to prove (ii) in most circumstances. This leaves (iii) as the most interesting step.

In practice the family $\mathcal{F}$ is usually of rank 3 and is obtained as follows. Prove the existence of a large subgroup $G_{1}$ of $G$ and consider the permutation representation of $G$ on the coset space $G / G_{1}$. Pick a selfpaired orbital $\theta$ for $G$ on $G / G_{1}$, so that the graph $\Delta$ determined by $\theta$ as in Example 3 is "nice". Now $G_{1}$ is the stabilizer of $x=G_{1}$ regarded as a point of $G / G_{1}$ and $G_{1}$ is transitive on the points adjacent to $x$ in $\Delta$. Pick such a point $y$ and let $G_{1}=G(\{x, y\})$ be the global stablizer of the pair 
$\{x, y\}$. Finally pick a large subgroup $G_{3}$ of $G$ such that graph $\Delta\left(G_{3}\right)$ with vertex set $x G_{3}$ and edge set $(x, y) G_{3}$ is "nice"; for example $G_{2}=\left(G_{1} \cap G_{2}\right)\left(G_{2} \cap G_{3}\right)$ and all triangles of $\Delta$ are fused into $\Delta\left(G_{3}\right)$ under $G$. (Call $\Delta\left(G_{3}\right)$ the residue of $G_{3}$.) Let $\mathcal{F}=\left(G_{1}, G_{2}, G_{3}\right)$. Then if $\Delta$ is simply connected, so is $\mathcal{C}(G, \mathcal{F})$, so we can work with $\Delta$.

The problem with this approach is that in order to prove simple connectivity of $\Delta$ given the methods known to me, the graph $\Delta$ must be very well behaved. This means that for each sporadic one must carefully choose the family $\mathcal{F}$ rather than making some uniform choice. As a result the approach is somewhat ad hoc and there is more effort expended in (i) than one would like. Here is an approach that might work for half of the sporadics and would be more uniform.

Assume $G$ satisfies Hypothesis $\mathcal{H}(w, L)$ for some $w$ and $L$, as do half the sporadics. Let $\Delta$ be the graph with vertex set $z^{G}$ and $z$ adjacent to $x$ iff $z \neq x \in Q$. The theory of so called large extraspecial subgroups (cf. [T], [A1],[A2]) shows that under some weak hypotheses, the edge set of this graph is a self paired orbital for $G$ on $G / H$ with $N_{G}(\langle z, x\rangle)$ the stabilizer of the edge $\{z, x\}$.

Question. Is the graph $\Delta$ simply connected?

Perhaps not. But the theory of large extraspecial subgroups guarentees the existence of a set $\mathcal{F}$ of canonical subgroups containing $Q$. If one considers the closure $C$ of all cycles of $\Delta G$-conjugate to cycles in the residues of members of $\mathcal{F}$, then the relation $\sim$ determined by $C$ should satisfy $\tilde{\pi}(\Delta, z) \cong \pi_{1}(\mathcal{C}(G, \mathcal{F}))$, so it would suffice to prove

(a) $\mathcal{A}(\mathcal{F})$ is determined up to isomorphism by $\mathcal{H}$, and

(b) $\tilde{\pi}_{1}(\Delta, z)=1$.

in order to prove $G$ is determined up to isomomorphism by $\mathcal{H}(w, L)$.

Problem 2. Complete the sketch above to prove the uniqueness of the 12 sporadic groups with a large extraspecial 2-subgroup.

\section{REFERENCES}

A1. M. Aschbacher, Overgroups of Sylow subgroups of sporadic groups, Memoirs AMS 342 (1986), 1-235.

A2. M. Aschbacher, "Sporadic Groups," Cambridge University Press, Cambridge, 1994.

AS. M. Aschbacher and Y. Segev, Extending morphisms of groups and graphs, Ann. Math. 135 (1992), 297-323.

T. F. Timmesfeld, Finite simple groups in which the generalized Fitting group of the centralizer of some involution is extra-special, Ann. Math. 107 (1978), 297-369. 